\title{
Microwave Assisted Synthesis, Characterization and Anti-Tubercular Activity of 4-Quinolylhydrazone
}

\section{Bisen Chandrakant V. ${ }^{1}$, ${ }^{*}$ Patle M. R. ${ }^{1}$, Rahangdale P.K. ${ }^{2}$}

1 D. B. Science College, Gondia, India

2 Bhavbhuti Mahavidyalaya, Amgaon, India

\section{ABSTRACT}

A series of 4-quinolylhydrazone derivatives was synthesized by reaction of 4-quinolylhydrazine and various substituted carboxaldehyde out of that most of the derivatives show significant antitubercular properties. The microwave assisted organic synthesis was applied to synthesize a series of 4-quinolylhydrazone derivatives. The characterizations of newly synthesized derivatives were done by modern analytical techniques like digital melting point apparatus, IR, NMR and mass spectroscopy.

Keywords: Mycobacterium tuberculosis, Hydrazone, Quinoline, Carboxaldehyde.

Article Info: Received 11 July 2019; $\quad$ Review Completed 17 August 2019; $\quad$ Accepted 21 August 2019; Available online 30 Aug 2019

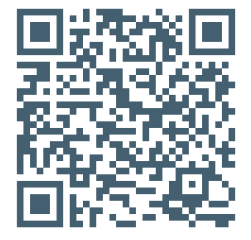

Cite this article as:

Bisen CV, Patle MR, Rahangdale PK, Microwave Assisted Synthesis, Characterization and Anti-Tubercular Activity of 4Quinolylhydrazone, Journal of Drug Delivery and Therapeutics. 2019; 9(4-A):95-97

http://dx.doi.org/10.22270/jddt.v9i4-A.3425

Patle M. R., D. B. Science College, Gondia, India

\section{Introduction:}

Tuberculosis (TB) is one of the most predominant infections in human beings and it has considerable contribution towards illness and death all around the world. Tuberculosis is caused by mycobacterium tuberculosis [1]. From previous research it is well known that quinolone is an important heterocyclic nucleus found in many natural as well as synthetic products having wide variety of pharmacological activities such as anti-TB [2], tyrokinase PDGF-RKT inhibiting agent [3], anticancer [4], antibacterial [5] and antiinflammatory [6]. The physicochemical study data of quinolone derivatives shows the potential antitubercular activity [7]. The literature study of some 4quinolylhydrazone derivatives unveiled significant activity (MIC=12.5-3.12 $\mu \mathrm{g} / \mathrm{ml}$ ) when compared to first line drugs such as ethambutol (MIC $=3.12 \mu \mathrm{g} / \mathrm{ml}$ ) [8]. With reference to this, in the search of new antituberculosis agents we proposed the synthesis of some quinolylhydrazones containing 4-hydrazinylquinoline moiety which was designed by using molecular modeling methods [9]. From literature survey it is observed that quinolylhydrazone moiety are pharmacologically very active, shows the activities like anti-inflammatory, antimicrobial and antitubercular [2]. The latest development in the field of organic chemistry is the microwave assisted organic synthesis (MAOS) [10], [11] which provides short reaction time and economic use of reagents through green approach [12].

\section{Experimental:}

The synthetic route for the preparation of 4quinolylhydrazone derivatives QH1 to QH8 is summarized in scheme 1 as below.<smiles></smiles>

Scheme 1: 4-quinolylhydrazine, corresponding carboxaldehydes, Ethanol, Microwave (MW) 10-15 min. 
A Mixture of 4-quinolylhydrazine (1 equivalent), and carboxaldehyde (1 equivalent) in absolute ethanol was irradiated with temperature assisted microwave oven at 180W for 10-15 min with intermittence. All the Chemicals used are of AR grade from Merck, India.

The completion of reaction is monitored by TLC. After conformation of completion of reaction by TLC the reaction mixture is cooled and diluted with water, so that respective hydrazones precipitated out from the reaction mixture. The product obtained was purified with column chromatography by using ethyl acetate and n-hexane to yield expected hydrazine derivatives. The purified derivatives were recrystallized using suitable organic solvent.

The molecules understudy are subjected to in-silico studies by using Datawarrior software for calculation of properties cLogP, cLogS, Total surface area, drug likeness and drug score to evaluate the therapeutic properties are summarized in table 1 as below.

Table1

\begin{tabular}{|c|c|c|c|c|c|c|c|}
\hline Hydrazones & Molecule Formula & $\begin{array}{c}\text { Total Molecular } \\
\text { weight }\end{array}$ & cLogS & H-acceptors & $\begin{array}{c}\text { H- } \\
\text { Donors }\end{array}$ & $\begin{array}{c}\text { Total } \\
\text { Surface } \\
\text { Area }\end{array}$ & Drug likeness \\
\hline QH1 & $\mathrm{C}_{16} \mathrm{H}_{13} \mathrm{~N}_{3}$ & 247.3 & -4.385 & 3 & 1 & 179.6 & -2.0672 \\
\hline $\mathrm{QH} 2$ & $\mathrm{C}_{16} \mathrm{H}_{12} \mathrm{ClN}_{3}$ & 281.745 & -5.121 & 3 & 1 & 195.0 & -0.2838 \\
\hline QH3 & $\mathrm{C}_{16} \mathrm{H}_{12} \mathrm{~N}_{4} \mathrm{O}_{2}$ & 292.297 & -4.845 & 6 & 1 & 203.3 & -9.0516 \\
\hline QH4 & $\mathrm{C}_{16} \mathrm{H}_{12} \mathrm{~N}_{4} \mathrm{O}_{2}$ & 292.297 & -4.845 & 6 & 1 & 203.3 & -7.0604 \\
\hline QH5 & $\mathrm{C}_{16} \mathrm{H}_{12} \mathrm{~N}_{4} \mathrm{O}_{2}$ & 292.297 & -4.845 & 6 & 1 & 203.3 & -11.868 \\
\hline QH6 & $\mathrm{C}_{17} \mathrm{H}_{15} \mathrm{~N}_{3} \mathrm{O}$ & 277.326 & -4.403 & 4 & 1 & 201.89 & -1.2243 \\
\hline QH7 & $\mathrm{C}_{16} \mathrm{H}_{13} \mathrm{~N}_{3} \mathrm{O}$ & 263.299 & -4.089 & 4 & 2 & 185.98 & -1.3401 \\
\hline QH8 & $\mathrm{C}_{17} \mathrm{H}_{13} \mathrm{~N}_{3} \mathrm{O}_{2}$ & 291.309 & -4.398 & 5 & 5 & 203.73 & -6.1263 \\
\hline
\end{tabular}

Furthermore the characterizations of hydrazine derivatives were established on the basis of spectral data analysis.

The synthesized 4-quinolylhydrazone derivatives with their percentage yield, melting point, clogP, drug score and biological activities are summarized in table 2 as below.

Table: 2

\begin{tabular}{|c|c|c|c|c|c|c|c|}
\hline Sr. No. & Hydrazones & Substitutions & $\%$ Yield & MP $\left({ }^{\circ} \mathrm{C}\right)$ & clogP & Drug Score & $\begin{array}{l}\text { Biological } \\
\text { Activity }\end{array}$ \\
\hline 1 & $\mathrm{QH} 1$ & $\mathrm{R}_{1}=\mathrm{R}_{2}=\mathrm{R}_{3}=\mathrm{H}$ & 71 & $223-224$ & 3.8788 & 0.39297 & 0.799 \\
\hline 2 & $\mathrm{QH} 2$ & $\mathrm{R}_{3}=\mathrm{Cl}, \mathrm{R}_{1}=\mathrm{R}_{2}=\mathrm{H}$ & 78 & $226-227$ & 4.4848 & 0.41254 & 0.786 \\
\hline 3 & $\mathrm{QH} 3$ & $\mathrm{R}_{1}=\mathrm{NO}_{2}, \mathrm{R}_{2}=\mathrm{R}_{3}=\mathrm{H}$ & 81 & $250-252$ & 2.9572 & 0.34878 & 0.780 \\
\hline 4 & $\mathrm{QH} 4$ & $\mathrm{R}_{2}=\mathrm{NO}_{2}, \mathrm{R}_{1}=\mathrm{R}_{3}=\mathrm{H}$ & 83 & $275-278$ & 2.9572 & 0.34904 & 0.790 \\
\hline 5 & $\mathrm{QH} 5$ & $\mathrm{R}_{3}=\mathrm{NO}_{2}, \mathrm{R}_{1}=\mathrm{R}_{2}=\mathrm{H}$ & 89 & $216-218$ & 2.9572 & 0.34874 & 0.786 \\
\hline 6 & $\mathrm{QH} 6$ & $\mathrm{R}_{3}=\mathrm{OCH}_{3}, \mathrm{R}_{1}=\mathrm{R}_{2}=\mathrm{H}$ & 76 & $146-148$ & 3.8088 & 0.43145 & 0.786 \\
\hline 7 & $\mathrm{QH} 7$ & $\mathrm{R}_{3}=\mathrm{OH}, \mathrm{R}_{1}=\mathrm{R}_{2}=\mathrm{H}$ & 84 & $220-221$ & 3.5331 & 0.45577 & 0.812 \\
\hline 8 & $\mathrm{QH} 8$ & $\begin{array}{c}\mathrm{R}_{3}=\mathrm{COOH}, \\
\mathrm{R}\end{array}=\mathrm{R}_{2}=\mathrm{H}$ & 80 & $221-223$ & 3.3639 & 0.36450 & 0.816 \\
\hline
\end{tabular}

\section{Spectral characterization of 4-quinolylhydrazone derivatives:}

\section{(Z)-2-benzylidene-1-(quinoline-4yl) hydrazine}

\section{$\left(\mathrm{QH1}-\mathrm{C}_{16} \mathrm{H}_{13} \mathrm{~N}_{3}\right)$ :}

${ }^{1} \mathrm{H}$ NMR (DMSO, $\left.\delta \mathrm{ppm}, \mathrm{TMS}\right) 8.18(\mathrm{~s}, 1 \mathrm{H}), 7.41-8.04(\mathrm{~d}, 4 \mathrm{H})$, 2.5(s1H), 7.39(t,1H), 7.44(d,1H), 7.51(t,1H), 7.59(d,1H). ${ }^{13} \mathrm{C}$ NMR -141, 150.3, 128, 128.9, 130.2, 125.8, 121.0. IR $\mathrm{cm}^{-1}$ $3070(\mathrm{CH}$ str), $1640(\mathrm{C}=\mathrm{N}$ str), $3320(\mathrm{NH}$ str $) \mathrm{MS} \mathrm{m} / \mathrm{z}$ 247(100\%), 248(18.4\%).

\section{(Z)-2-(4-chlorobenzylidene)-1-(quinoline-4yl)hydrazine}

(QH2-C $\left.\mathbf{1 1 6}_{16} \mathrm{H}_{12} \mathrm{ClN}_{3}\right):{ }^{1} \mathrm{H} \quad \mathrm{NMR} \quad$ (DMSO, $\left.\delta \mathrm{ppm}, \mathrm{TMS}\right)$ 8.17(d,1H), $\quad 8.04(\mathrm{~d}, 1 \mathrm{H}), \quad 8.50(\mathrm{~d}, 1 \mathrm{H}), \quad 7.30-7.60(\mathrm{~m}, 4 \mathrm{H})$, 3.95(s,1H). ${ }^{13} \mathrm{C}$ NMR $-143.1,150,132.1,136.2,129,146.9$, 121, 126.2 IR cm $\mathrm{cm}^{-1}-3070$ (CH str), $1640(\mathrm{C}=\mathrm{N}$ str), 3320(NH str), 782(C-Cl) MS m/z-281(100\%), 283(32.2\%).

\section{(Z)-2-(2-nitrobenzylidene)-1-(quinoline-4yl)hydrazine $\left(\mathrm{QH} 3-\mathrm{C}_{16} \mathrm{H}_{12} \mathrm{~N}_{4} \mathrm{O}_{2}\right)$ :}

${ }^{1} \mathrm{H}$ NMR (DMSO, $\delta$ ppm, TMS) 8.25(s,1H), 3.99(s,1H), 7.58.5(d,2H), 7.40-7.60, 8.60(s,1H). ${ }^{13} \mathrm{C}$ NMR -148.1, 144.2, $149.9,151.1,147.5,149.3,121.3,126.1 \mathrm{IR} \mathrm{cm}^{-1}-3070(\mathrm{CH}$ str), $1640\left(\mathrm{C}=\mathrm{N}\right.$ str), $3320\left(\mathrm{NH}\right.$ str), $1380\left(\mathrm{Ar}-\mathrm{NO}_{2}\right) \mathrm{MS} \mathrm{m} / \mathrm{z}-$ 292(100\%), 293(17.5\%).

ISSN: 2250-1177

\section{(Z)-2-(3-nitrobenzylidene)-1-(quinoline-4yl)hydrazine} (QH4- $\left.\mathrm{C}_{16} \mathrm{H}_{12} \mathrm{~N}_{4} \mathrm{O}_{2}\right)$ :

${ }^{1} \mathrm{H}$ NMR (DMSO, $\delta$ ppm, TMS) $8.20(\mathrm{~s}, 1 \mathrm{H}), 3.99(\mathrm{~s}, 1 \mathrm{H}), 1.49-$ 8.05(d,2H), $\left(8.5(\mathrm{~s}, 1 \mathrm{~h}){ }^{13} \mathrm{C}\right.$ NMR $-148.6,143,149.2,129.3$ $121,150.3,121.6 \mathrm{IR} \mathrm{cm}^{-1}-3070$ (CH str), $1640(\mathrm{C}=\mathrm{N}$ str), $3320\left(\mathrm{NH}\right.$ str), $1421\left(\mathrm{Ar}-\mathrm{NO}_{2}\right.$ str) MS m/z-292(100\%), 293(17.5\%).

\section{(Z)-2-(4-nitrobenzylidene)-1-(quinoline-4yl)hydrazine}

\section{$\left(\mathrm{QH} 5-\mathrm{C}_{16} \mathrm{H}_{12} \mathrm{~N}_{4} \mathrm{O}_{2}\right)$ :}

${ }^{1} \mathrm{H}$ NMR (DMSO, $\left.\delta \mathrm{ppm}, \mathrm{TMS}\right) \quad 8.0(\mathrm{~s}, 1 \mathrm{H}), \quad 8.01(\mathrm{t}, 2 \mathrm{H})$, $7.99(\mathrm{t}, 2 \mathrm{H}), \quad 8.54(\mathrm{~d}, 1 \mathrm{H}), \quad 7.49-8.05(\mathrm{~d}, 2 \mathrm{H})), \quad 6.41(\mathrm{~d}, 1 \mathrm{H}){ }^{13} \mathrm{C}$ NMR -150, 142.9, 121.3, 125.8, 128.8, 130, 130.2, 147.5 IR $\mathrm{cm}^{-1}-3070$ (CH str), $1640(\mathrm{C}=\mathrm{N}$ str), 3320(NH str), $1489(\mathrm{Ar}-$ $\mathrm{NO}_{2}$ str) MS m/z-292(100\%), 293(17.5\%).

\section{(Z)-2-(4-methoxybenzylidene)-1-(quinoline-}

\section{4yl)hydrazine}

(QH6- $\mathbf{C}_{17} \mathrm{H}_{15} \mathbf{N}_{3}$ O): ${ }^{1} \mathrm{H}$ NMR (DMSO, $\left.\delta \mathrm{ppm}, \mathrm{TMS}\right) 8(\mathrm{~s}, 1 \mathrm{H})$ $3.71(\mathrm{~s}, 3 \mathrm{H}), \quad 6.71(\mathrm{t}, 2 \mathrm{H}), \quad 7.54(\mathrm{t}, 2 \mathrm{H}), 3.99(\mathrm{~s}, 1 \mathrm{H}), \quad 7.67(\mathrm{~d}, 1 \mathrm{H})$, $7.40(\mathrm{t}, 1 \mathrm{~h}), 7.60(\mathrm{t}, 1 \mathrm{H}), 8.11(\mathrm{~d}, 1 \mathrm{H}){ }^{13} \mathrm{C}$ NMR $-60.1,162.9,114$, $126.2,130.4,115.2,129.3,125.4,121.5,143.2,147.6 \mathrm{IR} \mathrm{cm}^{-1}$ -3070 (CH str), $1640(\mathrm{C}=\mathrm{N} \mathrm{str}), 3320(\mathrm{NH} \mathrm{str}), 1266\left(\mathrm{Ar}-\underline{\mathrm{OCH}_{3}}\right)$ MS m/z-277(100\%), 278(19.5\%). 
(Z)-2-(4-hydroxybenzylidene)-1-(quinoline4yl)hydrazine

(QH7- $\left.\mathrm{C}_{16} \mathrm{H}_{13} \mathrm{~N}_{3} \mathrm{O}\right)$ :

${ }^{1} \mathrm{H}$ NMR (DMSO, $\delta$ ppm, TMS) $8(\mathrm{~s}, 1 \mathrm{H}), \quad 10.48(\mathrm{~s}, 1 \mathrm{H})$, $3.99(\mathrm{~s}, 1 \mathrm{H}), 6.50(\mathrm{~d}, 1 \mathrm{H}), 8.69(\mathrm{~d}, 1 \mathrm{H}), \quad 7.67(\mathrm{~d}, 1 \mathrm{H}), 7.40(\mathrm{t}, 1 \mathrm{H})$, $7.60(\mathrm{t}, 1 \mathrm{H}), 8.11(\mathrm{~d}, 1 \mathrm{H}){ }^{13} \mathrm{C}$ NMR $-160.7,115.9,143,121$ 125.5, 126.3, 130.3, 148.9 IR cm -1 $^{-3070(C H ~ s t r), ~} 1640(\mathrm{C}=\mathrm{N}$ str), 3320(NH str), 1216(Ar-프) MS m/z-263(100\%), 264(17.5\%).

\section{(Z)-2-(4-formylbenzylidene)-1-(quinoline-} 4yl)hydrazine

\section{$\left(\mathrm{QH} 8-\mathrm{C}_{17} \mathrm{H}_{13} \mathrm{~N}_{3} \mathrm{O}_{2}\right)$ :}

${ }^{1} \mathrm{H}$ NMR (DMSO, $\left.\delta \mathrm{ppm}, \mathrm{TMS}\right) 8(\mathrm{~s}, 1 \mathrm{H}), 13.26(\mathrm{~s}, 1 \mathrm{H})$, $3.99(\mathrm{~s}, 1 \mathrm{H}), 8.10(\mathrm{t}, 2 \mathrm{H}), 7.68(\mathrm{t}, 2 \mathrm{H}), \quad 7.67(\mathrm{~d}, 1 \mathrm{H}), \quad 7.40(\mathrm{t}, 1 \mathrm{H})$, 7.60(t,1H), 8.11(d,1H) ${ }^{13} \mathrm{C}$ NMR $-170.2,131.8,130.4,129.2$, 143, 150.1, 112.4, 129.3, 125.6, 130.4 IR cm-1 -3070(CH str), $1640(\mathrm{C}=\mathrm{N}$ str), $3320(\mathrm{NH}$ str), $1714(\mathrm{Ar}-\mathrm{COOH}) \mathrm{MS} \mathrm{m} / \mathrm{z}$ 291(100\%), 292(19.6\%).

\section{Results and Discussion:}

The series of 4-quinolylhydrazone derivatives from QH1 to QH8 had been synthesized using microwave assisted synthesis. Most of them show a good MIC value when compared with the first line drug Ethambutol with a very significant antitubercular activity. The TLC plate used is coated with alumina, column chromatography on silica gel (60-120mesh) was applied when required. 1H NMR spectra were recorded on VARIAN NMR spectrophotometer operating at $300 \mathrm{MHz}$, TMS is used as internal standard. IR spectrum recorded on Shimadzu IR Affinity-1S and mass spectra were recorded using water Micromass Q-Tof Mic.

\section{Conclusion:}

The series of 4-quinolylhydrazone based novel antitubercular agents were synthesized and studied by advanced and sophisticated instruments. As well as, these compounds are also subjected to in-silico studies for their therapeutic studies. These compounds shows drug score in the range of 0.34 to 0.45 and biological activity of about 0.80 against mycobacterium tuberculosis.

In this study it is observed that the hydrazone moiety having para substitution with withdrawing groups in benzylidene ring shows significant antitubercular activity studied by SAR study. In future this can be extended to synthesize more new derivatives and to study their practical application as potential antitubercular drugs.

\section{References:}

[1] "The global plan to stop TB," World Health Organization, 20112015.

[2] Lilienkampf A, Mao J, Wan B, Wang Y, Franzblau SG, Kozikowski AP, "Structure-activity relationships for a series of quinoline-based compounds active against replicating and nonreplicating Mycobacterium tuberculosis," vol. 52, 2009.

[3] Martin P. Maguire, Kimberly R. Sheets, Karen McVety, Alfred P. Spada, and Asher Zilberstein, "A New Series of PDGF Receptor Tyrosine Kinase Inhibitors: 3-Substituted Quinoline Derivatives," vol. 37, 1994.

[4] Denny WA, Wilson WR, Ware DC, Atwell GJ, Stevenson RJ, "Anti-cancer 2,3-dihydro-1H-pyrrolo[3,2-f]quinoline complexes of cobalt and chromium," 2006.

[5] Mahamoud A, Chevalier J, Davin-Regli A, Barbe J, Pagès JM "Quinoline derivatives as promising inhibitors of antibiotic efflux pump in multidrug resistant Enterobacter aerogenes isolates," vol. 7, 2006.

[6] Leatham PA, Bird HA, Wright V, Seymour D, Gordon A, "A double blind study of antrafenine, naproxen and placebo in osteoarthrosis," vol. 6, 1983.

[7] Beena and Diwan S. Rawat, "Antituberculosis Drug Research: A Critical Overview," Medicinal Research Reviews, pp. 693-764, 2013.

[8] André L.P.Candé, Marcelle de L.Ferreira, Karla C.Pais, Laura N.de F.Cardoso, Carlos R.Kaiser, Maria das Graças M.de O.Henriques, Maria C.S.Lourenço, Flávio A.F.M.Bezerra, Marcus V.N.de Souza, "Synthesis and antitubercular activity of 7chloro-4-quinolinylhydrazones derivatives," Bioorganic \& Medicinal Chemistry Letters, pp. 6272-6274, 2009.

[9] Ram Shankar Upadhayaya, Jaya Kishore Vandavasi, Nageswara Rao Vasireddy, Vivek Sharma, Shailesh S.Dixit, Jyoti Chattopadhyay., "Design, synthesis, biological evaluation and molecular modelling studies of novel quinoline derivatives against Mycobacterium tuberculosis," vol. 17, no. 7, 2009.

[10] Lindstroem p, Tierney J, Wathey B, Westman J, "Microwave assisted organic synthesis," vol. 57, 2001.

[11] Perreux L, Loupy A, "A tentative rationalization of microwave effects in organic synthesis according to the reaction medium, and mechanistic consideration," vol. 57, 2001.

[12] Varma R. S., "Solvent-free organic synthesis. using supported reagents and microwave irradiation," vol. 43, 1993.

[13] Sandra Gemma, Luisa Savini, Maria Altarelli, Pierangela Tripaldi, Luisa Chiasserini, Salvatore Sanna Coccone, Vinod Kumar, Caterina Camodeca, Giuseppe Campiani, Ettore Novellino, Sandra Clarizio, Giovanni Delogu, Stefania Butini, "Development of antitubercular compounds based on a 4quinolylhydrazone scaffold. Further structure-activity relationship studies," Bioorganic \& Medicinal Chemistry, pp. 6030-6072, 2009. 